\title{
SEARCHING FOR DIOPHANTINE QUINTUPLES
}

\author{
MIHAI CIPU AND TIM TRUDGIAN
}

\begin{abstract}
We consider Diophantine quintuples $\{a, b, c, d, e\}$. These are sets of distinct positive integers, the product of any two elements of which is one less than a perfect square. It is conjectured that there are no Diophantine quintuples; we improve on current estimates to show that there are at most $1.18 \cdot 10^{27}$ Diophantine quintuples.
\end{abstract}

\section{INTRODUCTION}

Define a Diophantine $m$-tuple as a set of $m$ integers $\left\{a_{1}, \ldots, a_{m}\right\}$ with $a_{1}<a_{2}<\ldots<$ $a_{m}$, such that $a_{i} a_{j}+1$ is a perfect square for all $1 \leq i<j \leq m$. Throughout the rest of this article we frequently refer to $m$-tuples, and not to Diophantine $m$-tuples.

It is conjectured that there are no quintuples - see [2, 13]. Successive authors (see, e.g., Table 1 in [16]) have reduced the bound on the possible number of quintuples. The best such published bound is $2.3 \cdot 10^{29}$ by Trudgian [16]. The purpose of this paper is to improve on this in the following theorem.

Theorem 1. There are at most $1.18 \cdot 10^{27}$ Diophantine quintuples.

In 92 we collect some ancillary results that aid the computational search for quintuples. In $\$ 3$ we obtain bounds on the relative sizes of elements in a quintuple. We use this in $\$ 4$ with results on linear forms of logarithms to obtain upper bounds on the second-largest element in a quintuple. In $\$ 5$ we examine some number-theoretic sums, which enable us to bound the total number of quintuples. We present two new arguments in $\$ 6$ that enable us to make a further saving, and ultimately to prove Theorem 1.

We are grateful to Adrian Dudek who suggested the asymptotic form in (9).

\section{DisCARDS}

It is known that every triple $\{a, b, c\}$ can be extended to a quadruple of a certain form. This is dubbed the 'regular' quadruple and is denoted as $\left\{a, b, c, d_{+}\right\}$. If a double or a triple cannot be extended to a non-regular quadruple, then it cannot be extended to a quintuple. We call such doubles or triples discards. The doubles $\{k, k+2\}$ [10] (see also [3]) are discards for $k \geq 1$. For an extensive list of discards, one may see [16, §2.1]. The following result allows us to recognise many discards.

Date: July 20, 2018.

2010 Mathematics Subject Classification. Primary 11D09; Secondary 11B37, 11J86, 11D45.

Key words and phrases. Diophantine $m$-tuples, Pell equations, linear forms in logarithms. 
Lemma 2.1. Let $\{a, b, c, d\}$ be a Diophantine quadruple with $a<b<c<d_{+}<d$.

- If $b<2 a$ then $b>21000$.

- If $2 a \leq b \leq 12 a$ then $b>130000$.

- If $b>12 a$ then $b>4001$.

Proof. The only difference between this lemma and Lemma 3.4 in [5] is the exclusion of the value $b=4001$ in the last case. Indeed, a pair $\{a, 4001\}$ with $12 a<4001$ cannot be extended because the equation $4001 a+1=r^{2}$ has unique integer solution $r<4001$, namely $r=4000$, which entails $a=3999$.

Lemma 2.2. ([5, Theorem 1.1]) Let $\{a, b, c, d, e\}$ be a quintuple with $a<b<c<d<e$ and put $g=\operatorname{gcd}(a, b)$. Then $b>3 a g$. If moreover $c>a+b+2 \sqrt{a b+1}$ then $b>$ $\max \left\{24 a g, 2 a^{3 / 2} g^{2}\right\}$.

Lemma 2.3. ([5, Theorem 1.3]) Let $\{a, b, c, d, e\}$ be a quintuple with $a<b<c<d<e$ and $c=a+b+2 \sqrt{a b+1}$. Then $b<a^{3}$ and $\operatorname{gcd}(b, c)=1$. In particular, at least one of $a, b$ is odd.

Examination of the relative size of entries in a quintuple has the following outcome.

Lemma 2.4. Any quintuple $\{a, b, c, d, e\}$ with $a<b<c<d<e$ must be of one of the types listed below:
(A) $4 a<b$ and $4 a b+b+a<c<b^{3 / 2}$,
(B) $4 a<b$ and $c=a+b+2 \sqrt{a b+1}$,
(C) $4 a<b$ and $c>b^{3 / 2}$,
(D) $b<4 a$ and $c=a+b+2 \sqrt{a b+1}$.

Proof. The claim follows from the previously cited results having in view [9, Lemma 4.2] and Subsection 2.2 in [4].

\section{Exploiting the connection with Pellian equations}

The entries in a quadruple are severely restricted in that they appear as coefficients of three generalized Pell equations that must have at least one common solution in positive integers. Each component of such a solution is obtained as a common term of two secondorder linearly recurrent sequences, giving rise to relations of the type $z=v_{m}=w_{n}$ for some positive integers $m$ and $n$. A key ingredient in the study of Diophantine sets is a relationship between the parameters $m, n$, and the values in the set in question.

Our next result is of this kind. It improves on several versions already in the literature — see, e.g., [4, 16, 17].

Proposition 3.1. Let $\{A, B, C, D\}$ be a quadruple with $A<B<C<D$ for which $v_{2 m}=w_{2 n}$ has a solution with $2 n \geq m \geq n \geq 2, m \geq 3$. Suppose that $A \geq A_{0}, B \geq B_{0}$, $C \geq C_{0}, B>\rho A$ for some positive integers $A_{0}, B_{0}, C_{0}$, and a real number $\rho>1$. Then

$$
m>\alpha B^{-1 / 2} C^{1 / 2} \text {, }
$$


where $\alpha$ is any real number satisfying both inequalities

$$
\begin{gathered}
\alpha^{2}+\left(1+\frac{1}{2} B_{0}^{-1} C_{0}^{-1}\right) \alpha \leq 4, \\
3 \alpha^{2}+\left(4 B_{0}\left(\lambda+\rho^{-1 / 2}\right)+2\left(\lambda+\rho^{1 / 2}\right) C_{0}^{-1}\right) \alpha \leq 4 B_{0},
\end{gathered}
$$

with $\lambda=\left(A_{0}+1\right)^{1 / 2}\left(\rho A_{0}+1\right)^{-1 / 2}$.

Moreover, if $C^{\tau} \geq \beta B$ for some positive real numbers $\beta$ and $\tau$ then

$$
m>\alpha \beta^{1 / 2} C^{(1-\tau) / 2} \text {. }
$$

Proof. We assume that $m \leq \alpha B^{-1 / 2} C^{1 / 2}$ and aim at establishing a contradiction if $\alpha$ is too small. We start from the congruence (see, e.g., [7, Lemma 4])

$$
\varepsilon A m^{2}+S m \equiv \varepsilon B n^{2}+T n \quad(\bmod 4 C) \quad \text { for some } \varepsilon= \pm 1,
$$

where $S=\sqrt{A C+1}$ and $T=\sqrt{B C+1}$. Since

$$
\left|A m^{2}-B n^{2}\right|<\max \left\{A m^{2}, B n^{2}\right\} \leq B m^{2} \leq \alpha^{2} C
$$

and

$$
\begin{aligned}
|S m-T n| & <\max \{S m, T n\} \leq T m \leq \alpha B^{-1 / 2} C^{1 / 2} \sqrt{B C+1} \\
& <\alpha B^{-1 / 2} C^{1 / 2}\left(B^{1 / 2} C^{1 / 2}+\frac{1}{2} B^{-1 / 2} C^{-1 / 2}\right) \leq \alpha\left(1+\frac{1}{2} B_{0}^{-1} C_{0}^{-1}\right) C,
\end{aligned}
$$

then, if $\alpha$ satisfies (1), the congruence (3) becomes the equality $A m^{2}-B n^{2}=\varepsilon(T n-S m)$. Multiplication by $T n+S m$ followed by rearrangements results in the equality

$$
\left(B n^{2}-A m^{2}\right)(C+\varepsilon(T n+S m))=m^{2}-n^{2} .
$$

Note that $B n^{2}=A m^{2}$ entails $m^{2}=n^{2}$, so that $A=B$ : a contradiction. Hence, for $m=n$ one necessarily has $C=T n+S m$, while for $m>n$ one finds that $B n^{2}-A m^{2}$ divides the positive integer $m^{2}-n^{2}$, so that $m^{2}-n^{2} \geq\left|A m^{2}-B n^{2}\right|$. This gives the following inequality

$$
\frac{m^{2}}{n^{2}} \geq \frac{B+1}{A+1}
$$

Having in view the lower bounds for $A$ and $B$, we obtain

$$
\frac{m^{2}}{n^{2}}>\frac{\rho A+1}{A+1} \geq \frac{\rho A_{0}+1}{A_{0}+1}=\frac{1}{\lambda^{2}} .
$$

From (4), $m \leq 2 n$, and the definitions of $S$ and $T$, we conclude that

$$
\begin{aligned}
C & \leq T n+S m+m^{2}-n^{2}<\lambda m \sqrt{B C+1}+m \sqrt{A C+1}+\frac{3}{4} m^{2} \\
& \leq \frac{3}{4} \alpha^{2} B^{-1} C+\alpha B^{-1 / 2} C^{1 / 2}\left(\lambda \sqrt{B C+1}+\sqrt{\rho^{-1} B C+1}\right) \\
& <\frac{3}{4} \alpha^{2} B^{-1} C+\alpha C\left(\lambda\left(1+\frac{1}{2} B^{-1} C^{-1}\right)+\rho^{-1 / 2}\left(1+\frac{1}{2} \rho B^{-1} C^{-1}\right)\right) \\
& \leq \frac{3}{4} \alpha^{2} B_{0}^{-1} C+\alpha C\left(\lambda\left(1+\frac{1}{2} B_{0}^{-1} C_{0}^{-1}\right)+\rho^{-1 / 2}\left(1+\frac{1}{2} \rho B_{0}^{-1} C_{0}^{-1}\right)\right) .
\end{aligned}
$$


The last expression is at most $C$ if $\alpha$ satisfies the inequality (2), whence the first inequality in the conclusion of our proposition. The second one is readily obtained from what we have just proved and the hypothesis $C^{\tau} \geq \beta B$.

Lemma 3.1. If $\{a, b, c, d, e\}$ is a quintuple with $a<b<c<d<e$ then the following bounds for $m$ hold:
(A) $m>3.3022 d^{1 / 4}$,
(D) $m>1.0080 d^{1 / 3}$.
(B) $m>1.5002 d^{2 / 7}$,
(C) $m>2.0604 d^{3 / 10}$

Proof. This is an application of the result just proved for $(A, B, C)=(a, b, d)$ in cases (A)-(C) and for $(A, B, C)=(a, c, d)$ in the remaining case. We use Proposition 3.1 with carefully chosen values for parameters in ranges suggested by Lemmas [2.1 2.3, In [12] it was shown that the first hypothesis required in Proposition 3.1 is satisfied. It is also known that one has $d>4 a b c+a+b+c$ (see, for instance, the proof of Lemma 6 in [8]).

In case (A) Lemma 2.2 hints to consider separately values of $a$ less than 144 since then one has $B=b>\max \left\{24 a, 2 a^{3 / 2}\right\}=24 a=24 A$. A short computer search finds that $B_{0}=4095$ and $\frac{b}{a} \geq \frac{21001}{143}>146=: \rho$. Clearly we must put $A_{0}=1$. From

$$
C=d>4 a b c+a+b+c>(4 a b+1)(4 a b+a+b)>\left(16 a^{2}+4 a\right) b^{2}
$$

it follows that $\tau=1 / 2, \beta=\left(16 A_{0}^{2}+4 A_{0}\right)^{1 / 2}, C_{0}>3.35 \cdot 10^{8}$ are admissible choices. Both inequalities (11) and (2) are satisfied by $\alpha=1.56155$.

Still in case (A), when $a \geq 144$ one puts $A_{0}=144, B_{0}=4002$ (by Lemma 2.1), $\rho=24$ (see Lemma 2.2) $, \tau=1 / 2, \beta=\left(16 A_{0}^{2}+4 A_{0}\right)^{1 / 2}$, whence $C_{0}>5.32 \cdot 10^{12}$ and $\alpha=1.56155$.

Having in view Lemma 2.1, in case (B) we first examine the subcase $4 a<b \leq 12 a$. Then $B_{0}=130001$, which implies $A_{0}=10834$ and $\rho=4$. From

$$
c>b\left(1+12^{-1}+2 \cdot 12^{-1 / 2}\right)=\left(1+12^{-1 / 2}\right)^{2} B
$$

and $a^{3}>b$ it follows that

$$
C=d>(4 a b+1)(a+b+2 r)>4\left(1+12^{-1 / 2}\right)^{2} a b^{2}>4\left(1+12^{-1 / 2}\right)^{2} B^{7 / 3},
$$

so that $\tau=3 / 7, \beta=\left(2+3^{-1 / 2}\right)^{6 / 7}, C_{0}=5.68 \cdot 10^{12}$. For these choices it is readily obtained that $\alpha=0.9999$ is permissible.

The other possibility in case $(\mathrm{B})$ is to have $b>12 a$. Convenient values of parameters are $\rho=12, A_{0}=16$ (from $a^{3}>b>4000$ ), $B_{0}=4002, \tau=3 / 7, \beta=2^{6 / 7}, C_{0}=1.01 \cdot 10^{9}$. for which the same value $\alpha=0.9999$ works.

Case (C) is similar to case (A). Now, for $a \leq 143$ we see that we can take $A_{0}=1$, $B_{0}=4004, \rho=28$. As

$$
C>4 a b c>4 a b^{5 / 2}>4.05 \cdot 10^{9}=: C_{0},
$$

we further get $\tau=2 / 5, \beta=4^{2 / 5}$, whence again $\alpha=1.5615$. In the complementary subcase $a \geq 144$, admissible values are $A_{0}=144, B_{0}=4002, \rho=24, \tau=2 / 5, \beta=576^{2 / 5}$, $C_{0}=5.83 \cdot 10^{11}$. Plugging these specializations into Proposition 3.1 , we obtain the same value for $\alpha$. 
Finally, in case (D) we have $A=a<b / 3, B=c=a+b+2 \sqrt{a b+1}>\left(1+3^{1 / 2}\right)^{2} A$, $B \leq a+b+2 \sqrt{3^{-1} b(b-1)+1}<\left(1+3^{-1 / 2}\right)^{2} b$, and

$$
C=d>4 a b c>b^{2} c>\left(1+3^{-1 / 2}\right)^{-4} B^{3} .
$$

Therefore, $\rho=\left(1+3^{1 / 2}\right)^{2}, \tau=1 / 3$, and $\beta=\left(1+3^{-1 / 2}\right)^{-4 / 3}$. From $130001 \leq b<4 a$, we have $A_{0}=32501$, whence $B_{0}>292504$, and $C_{0}>4.04 \cdot 10^{15}$. From (1) and (2) we obtain $\alpha=1.3660$.

For future reference, the values used in the previous proof are given in Table 1 .

\begin{tabular}{l|rrrrrr} 
Type & \multicolumn{1}{|c}{$A_{0}$} & \multicolumn{1}{c}{$B_{0}$} & \multicolumn{1}{c}{$C_{0}$} & $\rho$ & \multicolumn{1}{c}{$\beta$} & $\tau$ \\
\hline (AI) & 1 & 4095 & $3.35 \cdot 10^{8}$ & 146 & $20^{1 / 2}$ & $1 / 2$ \\
(AII) & 144 & 4002 & $5.32 \cdot 10^{12}$ & 24 & $24 \cdot 577^{1 / 2}$ & $1 / 2$ \\
(BI) & 10834 & 130001 & $5.68 \cdot 10^{12}$ & 4 & $\left(2+3^{-1 / 2}\right)^{6 / 7}$ & $3 / 7$ \\
(BII) & 16 & 4002 & $1.01 \cdot 10^{9}$ & 12 & $2^{6 / 7}$ & $3 / 7$ \\
(CI) & 1 & 4004 & $4.05 \cdot 10^{9}$ & 28 & $4^{2 / 5}$ & $2 / 5$ \\
(CII) & 144 & 4002 & $5.83 \cdot 10^{11}$ & 24 & $576^{2 / 5}$ & $2 / 5$ \\
(D) & 32501 & 292504 & $4.04 \cdot 10^{15}$ & $\left(1+3^{1 / 2}\right)^{2}$ & $\left(1+3^{-1 / 2}\right)^{-4 / 3}$ & $1 / 3$
\end{tabular}

TABLE 1. Parameter values for various types of Diophantine quintuples.

The values of $\alpha$, and hence the bounds on $m$ in Lemma 3.1, rely on the computational bounds in Lemma 2.1. While it is tempting to extend these computations, such an extension would have almost no effect on the values of $\alpha$. Consider, for example, case (A): sending $B_{0}, C_{0}$ to infinity in (1) gives $\alpha^{2}+\alpha \leq 4$. Therefore the optimal value of $\alpha$ is $1.5615528 \ldots$, whereas we have $\alpha=1.56155$. Likewise, in case (D) the optimal value is $\frac{1}{2}(1+\sqrt{3})=1.366025 \ldots$, whereas we have 1.3660 . It seems that a new idea is needed to improve substantially on the lower bounds on $m$.

\section{EMPLOYING LINEAR FORMS IN THE LOGARITHM}

The lower bounds for the index $m$ given in the previous section can be complemented by inequalities derived from upper bounds for linear forms of logarithms of algebraic numbers. To this end, we apply the best result of which we are aware.

Theorem 4.1 (Aleksentsev). Let $\Lambda$ be a linear form in logarithms of $n$ multiplicatively independent totally real algebraic numbers $\alpha_{1}, \ldots \alpha_{n}$, with rational coefficients $b_{1}, \ldots, b_{n}$. Let $h\left(\alpha_{j}\right)$ denote the absolute logarithmic height of $\alpha_{j}$ for $1 \leq j \leq n$. Let $d$ be the degree of the number field $\mathcal{K}=\mathcal{Q}\left(\alpha_{1}, \ldots, \alpha_{n}\right)$, and let $A_{j}=\max \left(d h\left(\alpha_{j}\right),\left|\log \alpha_{j}\right|, 1\right)$. Finally, let

$$
E=\max \left(\max _{1 \leq i, j \leq n}\left\{\frac{\left|b_{i}\right|}{A_{j}}+\frac{\left|b_{j}\right|}{A_{i}}\right\}, 3\right) \text {. }
$$

Then

$$
\log |\Lambda| \geq-5.3 n^{-n+1 / 2}(n+1)^{n+1}(n+8)^{2}(n+5)(31.44)^{n} d^{2}(\log E) A_{1} \cdots A_{n} \log (3 n d) .
$$


We have used the first displayed equation on [1, p. 2] to define $E$ in (5): this makes our application easier. We apply Theorem 4.1 for $d=4, n=3$ and to

$$
\Lambda=j \log \alpha_{1}-k \log \alpha_{2}+\log \alpha_{3},
$$

with

$$
\alpha_{1}=S+\sqrt{A C}, \quad \alpha_{2}=T+\sqrt{B C}, \quad \alpha_{3}=\frac{\sqrt{B}(\sqrt{C} \pm \sqrt{A})}{\sqrt{A}(\sqrt{C} \pm \sqrt{B})},
$$

where the signs coincide.

For our purposes we do not need the exact values of $A_{j}$ and $E$ as defined in Theorem 4.1: decent estimates will suffice. To find these estimates we proceed as follows, keeping the notation and hypotheses of Proposition 3.1 and supposing additionally that $C \leq C_{1}$ for a certain integer $C_{1}$.

We begin by noting that one has

$$
\begin{aligned}
2 \log \alpha_{1} & <\log (4 A C+4) \leq \log \left(4 \rho^{-1}(B-1) C+4\right)<\log \left(4 \rho^{-1} B C\right) \\
& <\log \left(4 \rho^{-1} \beta^{-1} C^{1+\tau}\right)
\end{aligned}
$$

provided that $\rho A \leq B-1$. This clearly follows from $\rho A<B$ when $\rho$ is integer, as in cases (A)-(C). In case (D) we have $b \geq 3 a+1$, so that (cf. the proof of Lemma 3.1)

$$
B=c=a+b+2 \sqrt{a b+1}>1+\left(1+3^{1 / 2}\right)^{2} a=1+\rho A .
$$

In each of the cases $(A)-(D)$ we have $\beta \rho>4$, whence

$$
A_{1}<g_{1}\left(\beta, \rho, \tau, C_{1}\right) \log C,
$$

with

$$
g_{1}\left(\beta, \rho, \tau, C_{1}\right):=1+\tau+\frac{\log 4-\log (\beta \rho)}{\log C_{1}} .
$$

We readily obtain the following lower bound on $A_{1}$

$$
A_{1}>\log (4 A C)>g_{2}\left(A_{0}, C_{1}\right) \log C,
$$

with

Similar relations hold for $A_{2}$, namely

$$
g_{2}\left(A_{0}, C_{1}\right):=1+\frac{\log 4+\log A_{0}}{\log C_{1}} .
$$

$$
2 \log \alpha_{2}<\log (4 B C+4)<\log \left(4 \beta^{-1} C^{1+\tau}+4\right),
$$

which implies the upper bound

$$
A_{2}<g_{3}(\beta, \tau, e) \log C,
$$

where

$$
g_{3}(\beta, \tau, e):=1+\tau+\frac{\log 4+\log \left(\beta^{-1}+e^{-1-\tau}\right)}{\log e}
$$


and $e=C_{0}$ in the cases $(\mathrm{B}),(\mathrm{CI})$, and (D) (when $\beta<4$ ) and $e=C_{1}$ in the remaining cases (A) and (CII). An easily-derived lower bound for $A_{2}$ is

$$
A_{2}>g_{4}\left(B_{0}, C_{1}\right) \log C,
$$

with

The inequalities

$$
g_{4}\left(B_{0}, C_{1}\right):=1+\frac{\log 4+\log B_{0}}{\log C_{1}} .
$$

$$
\frac{\sqrt{B}}{\sqrt{A}} \cdot \frac{\sqrt{C}+\sqrt{A}}{\sqrt{C}-\sqrt{B}}>\frac{\sqrt{B}}{\sqrt{A}} \cdot \frac{\sqrt{C}+\sqrt{A}}{\sqrt{C}+\sqrt{B}}>1, \quad \frac{\sqrt{B}}{\sqrt{A}} \cdot \frac{\sqrt{C}-\sqrt{A}}{\sqrt{C}-\sqrt{B}}>1
$$

are obvious. The modulus of the fourth algebraic conjugate of $\alpha_{3}$ is also greater than 1 precisely when $\sqrt{C}(\sqrt{B}-\sqrt{A})>2 \sqrt{A B}$. This inequality holds whenever

$$
\rho B_{0}^{1-\tau}\left(\rho^{1 / 2}-1\right)^{2 \tau}>2^{2 \tau} .
$$

It is easy to check that (6) is satisfied in each of the cases (A)-(D). One now obtains

$$
A_{3}=4 \mathrm{~h}\left(\alpha_{3}\right)=\log \left(\frac{B^{2}(C-A)^{2}}{g}\right),
$$

where $g$ is the content of the polynomial $A^{2}(C-B)^{2} X^{4}+4 A^{2} B(C-B) X^{3}+2 A B(3 A B-$ $\left.A C-B C-C^{2}\right) X^{2}+4 A B^{2}(C-A) X+B^{2}(C-A)^{2}$. Since $g$ is at most the smallest of the coefficients, which is $4 A^{2} B(C-B)$, one has

$$
\log \left(\frac{B(C-A)^{2}}{4 A^{2}(C-B)}\right) \leq A_{3} \leq \log \left(B^{2}(C-A)^{2}\right) .
$$

Note that $B(C-A)<\beta^{-1} C^{1+\tau}$ readily implies

$$
A_{3}<g_{5}(\beta, \tau, f) \log C,
$$

with

$$
g_{5}(\beta, \tau, f):=2+2 \tau-\frac{2 \log \beta}{\log f}
$$

and $f=C_{1}$ if $\beta>1$ and $f=C_{0}$ if $\beta<1$. A lower bound for $A_{3}$ is obtained with the help of the inequalities

$$
A_{3} \geq \log \left(\frac{B(C-A)^{2}}{4 A^{2}(C-B)}\right)>\log \left(\frac{\beta \rho^{2} C^{1-\tau}\left(1-A_{0} C_{1}^{-1}\right)^{2}}{4\left(1-\rho A_{0} C_{1}^{-1}\right)}\right),
$$

which entail

$$
A_{3}>g_{6}\left(\beta, \rho, \tau, A_{0}, C_{1}\right) \log C
$$

where

$$
g_{6}\left(\beta, \rho, \tau, A_{0}, C_{1}\right):=1-\tau+\frac{\log \left(\frac{1}{4} \beta \rho^{2}\right)+2 \log \left(1-A_{0} C_{1}^{-1}\right)-\log \left(1-4 C_{1}^{-1}\right)}{\log C_{1}} .
$$

On noting that for all relevant values of parameters one has $g_{2}\left(A_{0}, C_{1}\right)<g_{4}\left(B_{0}, C_{1}\right)$ and using the inequality $g_{2}>g_{6}$ (which follows, for $C_{1}>10^{12}$, from $16 C_{1}^{2 \tau}\left(1-\beta^{-1}\right)>\beta \rho^{2}$ 
if $\beta>1$ and from $16\left(1+3^{-1 / 2}\right)^{8 / 3} C_{1}^{2 / 3}\left(1-\rho A_{0} C_{1}^{-1}\right)>\rho^{3}$ in case (D)) as well as the known relation $k \leq j$, we find that we may take

$$
E \leq \frac{2 j}{g_{6}\left(\beta, \rho, \tau, A_{0}, C_{1}\right) \log C_{0}}
$$

Hence, Theorem 4.1 yields the following corollary.

\section{Corollary 4.2.}

$$
-\log \Lambda \leq 1.5013 \cdot 10^{11} g_{3} g_{5}\left(2 \log \alpha_{1}\right)\left(\log ^{2} C\right) \log \left(\frac{2 j}{g_{6} \log C_{0}}\right) .
$$

Corollary 4.2 bounds $\Lambda$ from below; we can bound $\Lambda$ from above using Eq. (4.1) in [12], which states that

$$
0<\Lambda<\frac{8}{3} A C \alpha_{1}^{-2 j}
$$

Comparison with Corollary 4.2 gives the main result of this section.

\section{Proposition 4.3.}

$$
j<1.50131 \cdot 10^{11} g_{3} g_{5}\left(\log ^{2} C\right) \log \left(\frac{2 j}{g_{6} \log C_{0}}\right) .
$$

Set $j=2 m$ in Proposition 4.3 and use Lemma 3.1 with the values given in Table 1 and $C_{1}=10^{72.188}$ in all cases, as per [4, Theorem 1.2]. We thus get a new upper bound on $d$ that we take as $C_{1}$ in a new iteration of this procedure. Slightly better bounds result by taking much higher $C_{0}$ (just below the value for $C_{1}$ considered in the same iteration). We record our computations in the following theorem.

Theorem 2. If $\{a, b, c, d, e\}$ is a quintuple with $a<b<c<d<e$ then the following bounds for d hold:
(A) $d<10^{67.859}<7.228 \cdot 10^{67}$
(C) $d<10^{56.528}<3.373 \cdot 10^{56}$,
(B) $d<10^{60.057}<1.141 \cdot 10^{60}$,
(D) $d<10^{51.416}<2.603 \cdot 10^{51}$.

We close this section with a remark concerning the size of the smallest entry in a quintuple. Although it has no immediate bearing on the next section, further improvements on $d$ should enable future researchers to enumerate all possible triples. Recording the maximal size of $a$ should aid this goal.

Proposition 4.4. The only quintuples that could arise from case (A) are those in which $a<7.4 \cdot 10^{7}$.

Proof. The triples in case (A) must satisfy $b^{3 / 2}>c>4 a b+b+a$, so that, in particular $a<b^{1 / 2} / 4$. Some quick computations give that for $A_{0}=7.4 \cdot 10^{7}$ one obtains $d<6.1 \cdot 10^{50}$. From $d>4 a b c>16 a^{2} b^{2}>\left(16 a^{2}\right)^{3}$ it then follows $a<7.29 \cdot 10^{7}$, a contradiction. 


\section{Bounding the total Number of Quintuples}

In this section we combine the methods of [4] and [16] in bounding certain arithmetical sums. Let

$$
E(x)=\sum_{n=1}^{x} 2^{\omega(n)}, \quad F(x)=\sum_{n=1}^{x} \frac{2^{\omega(n)}}{n}, \quad G(x)=\sum_{n=1}^{x} \frac{2^{\omega(2 n-1)}}{2 n-1},
$$

where all the sums are defined for real values of $x \geq 1$. Bounds for these sums can be used as in the proof of Lemma 4.4 in [4] to prove that

$$
\sum_{n=2}^{N} d_{H}\left(n^{2}-1\right) \leq 2 N G\left(\frac{H+1}{2}\right)+N G\left(\frac{H+4}{8}\right)+N G\left(\frac{H+2}{4}\right)+N G\left(\frac{H}{8}\right),
$$

where $d_{H}(n)$ counts the number of divisors of $n$ that do not exceed $H$. The function $d_{H}\left(n^{2}-1\right)$ arises naturally when considering the number of doubles $\{a, b\}$ satisfying certain restrictions.

The following lemma gives good bounds on $E(x)$ and $F(x)$.

Lemma 5.1 (Lemma 13 in [16]). For all $x \geq 1$ we have

$$
\begin{aligned}
& \sum_{n \leq x} \frac{2^{\omega(n)}}{n} \leq 3 \pi^{-2} \log ^{2} x+1.3948 \log x+0.4107+3.253 x^{-1 / 3}, \\
& \sum_{n \leq x} 2^{\omega(n)} \leq 6 \pi^{-2} x \log x+0.787 x+8.14 x^{2 / 3}-0.3762 .
\end{aligned}
$$

One can show, using Perron's formula and calculating residues, that

$$
\begin{aligned}
& E(x) \sim \frac{6}{\pi^{2}} x \log x+\frac{6}{\pi^{4}}\left(\pi^{2}(2 \gamma-1)-12 \zeta^{\prime}(2)\right) x, \\
& F(x) \sim \frac{3}{\pi^{2}} x \log x+\frac{12}{\pi^{4}}\left(\pi^{2} \gamma-6 \zeta^{\prime}(2)\right) x,
\end{aligned}
$$

where

$$
\frac{6}{\pi^{4}}\left(\pi^{2}(2 \gamma-1)-12 \zeta^{\prime}(2)\right)=0.78687 \ldots, \quad \frac{6}{\pi^{4}}\left(\pi^{2}(2 \gamma-1)-12 \zeta^{\prime}(2)\right)=1.39479 \ldots
$$

This shows that up to three decimal places, the bounds in Lemma 5.1 agree with the asymptotic expansions to the first two terms.

Similarly, one can calculate the asymptotic order of $G(x)$. For $\Re(s)>1$ we have

$$
\sum_{n=1}^{\infty} \frac{2^{\omega(n)}}{n^{s}}=\frac{\zeta^{2}(s)}{\zeta(2 s)}=\prod_{p}\left(1+\frac{2}{p^{s}}+\frac{2}{p^{2 s}}+\cdots\right) .
$$

We can remove the contribution of $p=2$ to yield

$$
\sum_{n=1, n \text { odd }}^{\infty} \frac{2^{\omega(n)}}{n^{s}}=\frac{1-2^{-s}}{1+2^{-s}} \frac{\zeta^{2}(s)}{\zeta(2 s)}
$$


whence, by Perron's formula we have

$$
\begin{aligned}
G(x)=\sum_{n \leq 2 x, n \text { odd }} \frac{2^{\omega(n)}}{n} & \sim \operatorname{Res}\left\{\frac{1-2^{-(s+1)}}{1+2^{-(s+1)}} \frac{\zeta^{2}(s+1)}{\zeta(2(s+1))} \frac{(2 x)^{s}}{s} ; \quad s=0\right\} \\
& \sim \frac{1}{\pi^{2}} \log ^{2} x+\frac{2}{3 \pi^{4}}\left\{\pi^{2}(6 \gamma+7 \log 2)-36 \zeta^{\prime}(2)\right\} \log x .
\end{aligned}
$$

Were one to use this in the proof of Lemma 4.4 in [4] one would have

$$
\sum_{n=2}^{N} d_{H}\left(n^{2}-1\right) \leq \frac{7 N}{\pi^{2}} \log ^{2} H+\ldots
$$

It would take considerable effort to furnish an explicit version of (10). Even if the lower order terms in (10) were negligible, one would only save a factor of two for the values of $H$ and $N$ used in our calculations. We have therefore not pursued this 1

We content ourselves with the bound for $G(x)$ as given in Lemma 4.3 in [4], namely

$$
G(x)<\frac{3}{2 \pi^{2}} \log ^{2} x+3.1227147 \log x+3.56851+\frac{0.525}{x} .
$$

Using this and (86) in (7) we follow the proof of Lemma 4.4 in [4] to prove

Lemma 5.2. Let $d_{H}(n)$ denote the number of positive integers e such that e $n$ and $e \leq H$. Then, for any $N \geq 2$ and $H \geq 1$ we have

$$
\sum_{n=2}^{N} d_{H}\left(n^{2}-1\right)<N\left\{\frac{9}{\pi^{2}} \log ^{2} H+11.1468 \log H-0.957+\frac{24 \log H}{\pi^{2} H}+\frac{44.14}{H}\right\} .
$$

We now proceed to examine the number of quintuples that could arise from each of the triples $(\mathrm{A})-(\mathrm{D})$.

5.1. Case (A). This is the most damaging case in our considerations. We have $r<$ $(d / 16)^{1 / 4}$, whence, by Theorem 2 we have $r<4.611 \cdot 10^{16}=R_{A}$. Using Lemma 5.2 we find that the number of doubles is at most

$$
\frac{1}{2} \sum_{r=3}^{R_{A}} d_{R_{A}}\left(r^{2}-1\right)<4.080 \cdot 10^{19}
$$

Since $b<(d / 20)^{1 / 2}<1.9011 \cdot 10^{33}$ we find that $b$ could have as many as 23 distinct prime factors. We find that the number of quintuples is therefore bounded by

$$
3 \cdot 4 \cdot 2^{24} \cdot 4.08 \cdot 10^{19} \leq 8.215 \cdot 10^{27} .
$$

Since the number of possible quintuples originating from case (A) is by far the largest, we devote $\$ 6$ to reducing this number slightly.

\footnotetext{
${ }^{1}$ We note that Dudek [6] has recently shown that $\sum_{n=2}^{N} d\left(n^{2}-1\right) \sim 6 \pi^{-2} N \log ^{2} N$.
} 
5.2. Case (B). Since $b>4 a$ we have $b>2 r$, whence $c>4 r+a$. Since $d>4 a b c$ this shows that $d>4\left(r^{2}-1\right)(4 r+2)>16 r^{3}$. From Theorem 2 we therefore have $r \leq 4.147 \cdot 10^{19}=R_{B}$. By Lemma 5.2 the number of doubles $\{a, b\}$ is at most

$$
\frac{1}{2} \sum_{r=3}^{R_{B}} d_{R_{B}}\left(r^{2}-1\right)<4.91 \cdot 10^{22} .
$$

Since there are at most four ways of extending a quadruple to a quintuple we find that the total number of quintuples is bounded above by

$$
2.0 \cdot 10^{23} \text {. }
$$

5.3. Case (C). We proceed as in case 2(iii) in [16]. We consider the cases $a>\eta$ and $a \leq \eta$ and optimise over $\eta$. In the former case, we have $d>4 a b c>4 \eta b^{5 / 2}$ so that $b<(d /(4 \eta))^{2 / 5}:=N_{3 a}$. Hence, by Lemma 3.3 in [9], the number of quintuples is at most

$$
\frac{N_{3 a}}{6}\left(\log N_{3 a}+2\right)^{3} \cdot 8 \cdot 5 \cdot 4 .
$$

When $a \leq \eta$, we have $b<(d /(4 a))^{2 / 5}$ so that $r^{2}=a b+1<a(d /(4 a))^{2 / 5}+1$. Thus

$$
r<\sqrt{1+\left(\frac{\eta^{3} d^{2}}{16}\right)^{1 / 5}}=N_{3 b} .
$$

We apply Lemma 5.2 with $H=\eta$ and $N=N_{3 b}$. Since $b<(d / 4)^{2 / 5}<2.35 \cdot 10^{22}$ we have $\omega(b) \leq 17$. Following the proof in [9] we deduce that the number of quintuples is at most

$$
4 \cdot 2^{17} \cdot 5 \cdot 4 \cdot N_{3 b}\left(\frac{9}{\pi^{2}} \log ^{2} \eta+11.1468 \log \eta-0.957+\frac{24 \log \eta}{\pi^{2} \eta}+\frac{44.14}{\eta}\right) .
$$

We find that we can minimise the maximum of (13) and (14) at $\eta=6.76 \cdot 10^{10}$. Hence the number of quintuples is at most

$$
2.41 \cdot 10^{22}
$$

5.4. Case (D). We have $b<(4 d / 9)^{1 / 3}$ so that, by Theorem 2, we have $b<1.05 \cdot 10^{17}=$ $R_{D}$. The number of doubles $\{a, b\}$ is therefore bounded by $2 \sum_{b=4}^{R_{D}} 2^{\omega(b)}$. We use this and Lemma 5.1 to prove that the number of quintuples is at most

$$
2.07 \cdot 10^{19} \text {. }
$$

\section{IMPROVEMENTS TO CASE (A)}

Here we investigate two methods. The first, in $\$ 6.1$, reduces the bound on $\omega(b)$ from 23 to 22 , thereby saving a factor of 2 in the estimate recorded in (111). The second, in $\$ 6.2$, splits up the sum over $b$ with $\omega(b)$ held constant. This saves a factor of about 3.58. 
6.1. Removing one prime factor from $b$. Let $\left(p_{n}\right)_{n \in \mathbb{N}}$ denote the sequence of prime numbers, and consider those $b$ satisfying

$$
b_{0}:=\prod_{i=1}^{23} p_{i} \approx 2.67 \cdot 10^{32} \leq b<1.9011 \cdot 10^{33}, \quad \omega(b)=23 .
$$

We aim at enumerating all such $b$ in (17). We shall show that none of these values of $b$ can appear as the second-smallest element of a quintuple. This then shows that $\omega(b) \leq 22$, and leads immediately to a saving of a factor of 2 in (11).

Suppose $\{a, b, c, d, e\}$ is a quintuple. In case (A), Theorem 2 gives the bound $d<U D:=$ $10^{67.859}$. When $b$ is restricted as in (17) we find that 2 divides $b$, since, if not, the smallest $b$ can be is $\prod_{i=1}^{23} p_{i} / 2 \cdot p_{24}>1.18 \cdot 10^{34}$. Continuing in this way we find that $2,3,5,7,11$ must all divide $b$.

From $4 a(4 a+1) b^{2}<U D$ it then follows $a \leq 7$. Moreover, as the corresponding $r$ is odd, $a b$ is a multiple of 8 , whence $b \equiv 0(\bmod 8)$ for odd $a$ and $b \equiv 0(\bmod 4)$ for $a \equiv 2$ (mod 4). Hence, each such $b$ is obtained from $b_{1}=b_{1}(a)$ by replacing $v$ of its factors $p_{6}$, $\ldots, p_{23}$ by other $v$ primes $p_{k_{1}}, \ldots, p_{k_{v}}$, where $24 \leq k_{1}<\cdots<k_{v}$, and then multiplying by some positive integer $q$ such that the result is at most

$$
U B=U B(a, U D):=U D^{1 / 2}\left(16 a^{2}+4 a\right)^{-1 / 2} .
$$

Here $b_{1}(a)=4 b_{0}$ if $a$ is odd, $b_{1}(a)=2 b_{0}$ if $a=2,6$, and $b_{1}(a)=b_{0}$ otherwise.

We now present a detailed exposition of the idea sketched above. All computations have been performed with GP scripts [15]. Clearly, the maximal $v$ is determined from the condition

$$
\frac{p_{24} p_{25} \cdots p_{23+v}}{p_{23} p_{22} \cdots p_{24-v}}<\frac{U B}{b_{1}}
$$

A short computer search gives $v=3$ for $a=2$ or 4 ; $v=2$ for $a=1 ; v=0$ for the other values $a \leq 7$.

Next for each $u=1,2, \ldots, v$ we look for the largest index $K=K(u)$ satisfying

$$
\frac{p_{24} p_{25} \cdots p_{22+u} p_{K}}{p_{23} p_{22} \cdots p_{24-u}}<\frac{U B}{b_{1}}
$$

and the smallest $J$ verifying

$$
\frac{p_{24} p_{25} \cdots p_{23+u}}{p_{23} p_{22} \cdots p_{25-u} p_{J}}<\frac{U B}{b_{1}}
$$

After that we determined all integers $24 \leq k_{1}<\cdots<k_{u} \leq K$ and $23 \geq j_{1}>\cdots>j_{u} \geq J$ such that

$$
\frac{p_{k_{1}} p_{k_{2}} \cdots p_{k_{u}}}{p_{j_{1}} p_{j_{2}} \cdots p_{j_{u}}}<\frac{U B}{b_{1}}
$$

Each such tuple $\left(k_{1}, \ldots, k_{u}, j_{1}, \ldots, j_{u}\right)$ gives rise to

$$
\left\lfloor\frac{U B p_{j_{1}} p_{j_{2}} \cdots p_{j_{u}}}{b_{1} p_{k_{1}} p_{k_{2}} \cdots p_{k_{u}}}\right\rfloor
$$

candidates for the largest entry in a Diophantine couple $\{a, b\}$. 
Since the bound $U D=10^{67.859}$ found in case (A) entails $U B(a, U D)<10^{33.9295}\left(16 a^{2}+\right.$ $4 a)^{-1 / 2}$, for $1 \leq a \leq 7$ one has

$$
\frac{U B(a, U D)}{b_{1}(a)} \leq \frac{U B(4, U D)}{b_{1}(4)}<2 .
$$

Therefore, the multiplier $q$ mentioned above must be equal to 1 .

For each value of $b$ identified using the above method, we are able to show easily that there is no corresponding quadruple. This shows that $\omega(b) \leq 22$. In theory there is nothing stopping us from playing this trick again. However, when we search for $\omega(b)=22$ we find that we could have over four thousand primes dividing $b$. This appears to be orders of magnitude harder than the $\omega(b)=23$ case.

6.2. Bounding $b$ in different ranges. We have $a b+1=r^{2}$. Note first that $d\left(r^{2}-1\right)$ is even (it is odd if and only if $r^{2}-1=s^{2}$ which implies that $(r+s)(r-s)=1-$ a contradiction). Since $d\left(r^{2}-1\right)$ counts the number of divisors of $r^{2}-1$ it follows that $\frac{1}{2} d\left(r^{2}-1\right)$ counts the number of pairs of divisors $\{a, b\}$ with $a<b$. Now each $a$ corresponds with exactly one $b$ (and hence one pair corresponds with exactly one value of $a$ ): therefore $\frac{1}{2} d\left(r^{2}-1\right)$ is actually counting the divisors $a$. Furthermore, note that

$$
r^{2}-1=a b>a^{2} .
$$

Therefore $\frac{1}{2} d\left(r^{2}-1\right)$ is actually counting all those $a$ with $a<\sqrt{r^{2}-1}$. Whence for a fixed $r$ we wish to count

$$
\frac{1}{2} d_{\sqrt{r^{2}-1}}\left(r^{2}-1\right)
$$

If $r \leq R$ then summing over $r$ shows that the number of pairs $\{a, b\}$ is at most

$$
\frac{1}{2} \sum_{r=3}^{R} d_{\sqrt{r^{2}-1}}\left(r^{2}-1\right) \leq \frac{1}{2} \sum_{r=3}^{R} d_{r}\left(r^{2}-1\right)<\frac{1}{2} \sum_{r=3}^{R} d_{R}\left(r^{2}-1\right) .
$$

Now, we can make a slight improvement on (19). Since, for case (A) quadruples we have $b>4 a$, we can improve on (18) to show that $r^{2}-1=a b>4 a^{2}$. Therefore, we amend (19) to show that the total number of pairs is at most

$$
\frac{1}{2} \sum_{r=3}^{R} d_{R / 2}\left(r^{2}-1\right)
$$

One can go further than this. Let $N(\alpha, \beta)$ be the number of quintuples with $\alpha a<b \leq$ $\beta a$, for some $\beta>\alpha \geq 4$. It then follows that for integers $m_{i}$ satisfying $4=m_{0}<m_{1}<$ $\ldots<m_{k}$ the total number of quintuples is bounded above by

$$
N\left(4, m_{1}\right)+N\left(m_{1}, m_{2}\right)+\cdots+N\left(m_{k-1}, m_{k}\right)+N\left(m_{k}, \infty\right),
$$

where $N\left(m_{k}, \infty\right)$ means all those pairs $\{a, b\}$ such that $b>m_{k} a$. With the exception of $N\left(m_{k}, \infty\right)$, each number is of the form $N\left(m_{j}, m_{j+1}\right)$. 
Take $m_{j} a<b \leq m_{j+1} a$. Since $d>4 a b(4 a b+a+b)>16 a^{2} b^{2}>16 b^{4} /\left(m_{j+1}\right)^{2}$ we have

$$
b<\frac{d^{1 / 4}\left(m_{j+1}\right)^{1 / 2}}{2} \text {. }
$$

We also have

$$
r^{2}-1=a b>m_{j} a^{2} \Rightarrow a<R / \sqrt{m_{j}} .
$$

By taking $m_{j}$ large we ensure that the bound on $a$ in (21) is small. We now look at $\omega(b)$ for $b$ satisfying (20). We want to choose $m_{j+1}$ to be as large as possible such that we do not increase $\omega(b)$. For example, when $j=0$ we are considering $4 a<b \leq m_{1} a$. We find, using $d \leq 7.228 \times 10^{67}$, that $\omega(b) \leq 14$ provided that $m_{1} \leq 177$. Also, for $m_{2}$ we find that we can take $m_{2} \leq 499686$ and still ensure that $\omega(b) \leq 15$. We continue in this way, contenting ourselves with estimates on $m_{j}$ that are accurate to one decimal place. We find, using Mathematica [14, that we may take

$$
\left(m_{3}, m_{4}, m_{5}, m_{6}, m_{7}, m_{8}\right)=\left(1.7 \cdot 10^{9}, 6.4 \cdot 10^{12}, 2.9 \cdot 10^{16}, 1.4 \cdot 10^{20}, 7.8 \cdot 10^{23}, 4.8 \cdot 10^{27}\right) \text {. }
$$

We know, from $\$ 6.1$, that there are at most 22 distinct prime factors of $b$. Therefore we have that the number of quintuples is at most

$$
3 \cdot 2\left(2^{15} \sum_{r=3}^{R} d_{R / 2}\left(r^{2}-1\right)+2^{16} \sum_{r=3}^{R} d_{R / \sqrt{177}}\left(r^{2}-1\right)+\cdots+2^{23} \sum_{r=3}^{R} d_{R / \sqrt{4 \cdot 8 \cdot 10^{27}}}\left(r^{2}-1\right)\right) .
$$

We find that the above is no more than

$$
1.177 \cdot 10^{27} \text {. }
$$

Using (12), (15), (16) and (22) we complete the proof of Theorem 1 .

\section{REFERENCES}

[1] Y. M. Aleksentsev, The Hilbert polynomial and linear forms in the logarithms of algebraic numbers, Izv. Math. 72 (2008), 1063-1110.

[2] J. Arkin, V. E. Hoggatt, E. G. Straus, On Euler's solution of a problem of Diophantus, Fibonacci Quart. 17 (1979), 333-339.

[3] Y. Bugeaud, A. Dujella, M. Mignotte, On the family of Diophantine triples $\left\{k-1, k+1,16 k^{3}-4 k\right\}$, Glasgow Math. J. 49 (2007), 333-344.

[4] M. Cipu, Further remarks on Diophantine quintuples, Acta Arith., 168 (2015), 201-219.

[5] M. Cipu, A. Filipin, Y. Fujita, Bounds for Diophantine quintuples II, preprint, 2015.

[6] A. Dudek, On the number of divisors of $n^{2}-1$, Bull. Aust. Math. Soc., to appear.

[7] A. Dujella, An absolute bound for the size of Diophantine m-tuples, J. Number Theory 89 (2001), 126-150.

[8] A. Dujella, There are only finitely many Diophantine quintuples, J. Reine Angew. Math. 566 (2004), 183-224.

[9] C. Elsholtz, A. Filipin, Y. Fujita, On Diophantine quintuples and D(-1)-quadruples, Monats. Math. 175 (2014), 227-239.

[10] Y. Fujita, The extensibility of Diophantine pairs $\{k-1, k+1\}$, J. Number Theory 128 (2009), $322-353$.

[11] Y. Fujita, Any Diophantine quintuple contains a regular Diophantine quadruple, J. Number Theory 129 (2009), 1678-1697. 
[12] Y. Fujita, The number of Diophantine quintuples, Glas. Mat. Ser. III 45 (2010), 15-29.

[13] P. E. Gibbs, Computer Bulletin 17 (1978), 16.

[14] Wolfram Research, Inc., Mathematica, Version 10.1, Champaign, IL (2015).

[15] The PARI Group, PARI/GP, version 2.3.5, Bordeaux, 2010, available from http://pari.math.u-bordeaux.fr/.

[16] T. S. Trudgian, Bounds on the number of Diophantine quintuples, J. Number Theory 157 (2015), 233-249.

[17] W. Wu, B. He, On Diophantine quintuple conjecture, Proc. Japan Acad. Ser. A Math. Sci. 90 (2014), $84-86$.

Simion Stoilow Institute of Mathematics of the Romanian Academy, Research unit nr. 5, P.O. Box 1-764, RO-014700 Bucharest, Romania

Email: Mihai.Cipu@imar.ro

Mathematical Sciences Institute, The Australian National University, Canberra, Australia Email: Timothy.Trudgian@anu.edu.au 